\title{
The detectability of WCDA to TeV-sources
}

\section{Yingying Guo*}

Institute of High Energy Physics, Chinese Academy of Sciences, Beijing, 100049, China

E-mail: yyguo@ihep.ac.cn

\section{Xiaochuan Chang}

Institute of High Energy Physics, Chinese Academy of Sciences, Beijing, 100049, China

\section{Hongbo Hu}

Institute of High Energy Physics, Chinese Academy of Sciences, Beijing, 100049, China

\section{Zhiguo Yao}

Institute of High Energy Physics, Chinese Academy of Sciences, Beijing, 100049, China

\section{On behalf of the LHAASO Collaboration}

The Large High Altitude Air Shower Observatory (LHAASO) as a new generation of cosmic ray experiment with unprecedented sensitivity is under construction, and the Water Cherenkov Detector Array (WCDA) is one major component aiming at $\mathrm{TeV} \gamma$ rays. In order to sufficiently understand the prospect of searching for $\mathrm{TeV} \gamma$-ray sources with WCDA, one-year observing ability of WCDA to TeV $\gamma$-ray sources in TeVCat is presented in this paper. There are 56 out of $128 \gamma$-ray sources in the $45^{\circ}$ view of WCDA would be observed at the middle energy of $1 \mathrm{TeV}$. Most of them are galactic sources, and the extragalactic sources are mainly active galactic nuclei (AGNs).

36th International Cosmic Ray Conference -ICRC2019-

July 24th - August 1st, 2019

Madison, WI, U.S.A.

\footnotetext{
${ }^{*}$ Speaker.
} 


\section{Introdution}

The very high energy (VHE, $>100 \mathrm{GeV}$ ) gamma rays plays a key role in allowing us to comprehend puzzles in modern astrophysics and cosmology, such as the origin of galactic and extragalactic Cosmic Rays(CRs), the acceleration and radiation process in the violent environment, e.g. in the shock of SNRs, in the outflow of active galactic nuclei and pulsar winds. Besides, it may contribute to cosmological issues via confining the annihilation cross section of dark matter like WIMPs and searching for Lorentz violation, etc.

Both the space-borne and ground based technique are used to detect the very high energy (VHE) $\gamma$ rays. The Fermi Large Area Telescope (LAT), representing the space-borne observatories, have found thousands of $\gamma$-ray sources in $\mathrm{GeV}$ band. Because of the low $\gamma$-ray flux at higher energies make space detectors insensitive and expensive compared with ground-based observatories. The traditional extensive air shower(EAS) array, like Tibet AS $\gamma$ and ARGO-YBJ are ineffective in distinguishing $\gamma$ rays from CRs, therefore it is not ideal for detecting $\gamma$ rays. Water Cherenkov technique is developed by Milagro [1] to observe the Cherenkov light emitted by secondary particles (both electromagnetic particles and Muons) going through the pure water, which shows a better $\gamma /$ proton discrimination compared with traditional EAS array. $\mathrm{TeV} \gamma$-ray surveys with Milagro and HAWC have been reported in [1] [2]. There are 39 sources, which have been detected by HAWC [3]. $\gamma$-ray signals at least $25 \mathrm{TeV}$, which are proposed to be produced by electrons with energies extending to at least several hundreds of $\mathrm{TeV}$ in a magnetic field about 16 micro-Gauss, have been reported in [4]. This discovery provides valuable probes of the particle acceleration mechanisms in jets. Compared to previous experiments, WCDA is larger. It implies that WCDA has great potential to detect more $\gamma$-ray sources.

In this paper we demonstrate the ability of WCDA to search for $\gamma$-ray sources. In Section 2 , WCDA is briefly introduced, and in Section 3 we describe the Fast simulation process. Finally, in Section 4, we predict the significance of point-like sources.

\section{The Water Cherenkov Detector Array}

The WCDA measures showers in a primary energy range from $\sim 100 \mathrm{GeV}$ to $20 \mathrm{TeV}$ and constitutes one important part of LHAASO that operates at coordinates of $29^{\circ} 21^{\prime} 31^{\prime \prime} \mathrm{N}, 100^{\circ} 08^{\prime} 15^{\prime \prime} \mathrm{E}$, at an altitude of $4410 \mathrm{~m}$ in Daocheng site, Sichuan province, P.R. China. The original design is a $90,000 \mathrm{~m}^{2}$ array which is divided into 4 subarrays with size of $150 \mathrm{~m} \times 150 \mathrm{~m}$. One upward-facing 8-inch photomultiplier tube (PMTs) is attached to the center of the bottom of each cell measuring $5 \mathrm{~m} \times 5 \mathrm{~m}$, and each subarray owns 900 PMTs. The time and amplitude of PMT signals would be used to reconstruct the arrival directions and core positions of primary particles. The prospect of WCDA reported in this paper is based on such configuration. It should be noted that the design has been modified and we will discuss the effect of such differences on our result in Section 5.

A simulation according to the original design mentioned above was completed and reported in paper[5]. This simulation tracks the most luminous gamma-ray source, Crab, and simulates $\gamma$-ray showers from Crab and the CR showers within $7^{\circ}$ radius around Crab. On the basis of this simulation, we adopt a criterion that the number of fired PMTs is more than 128, representing the $\gamma$ rays at the middle energy of $1 \mathrm{TeV}$. The effective area of $\gamma$ rays as a function of energy and zenith 
angle shows in Figure 1(a), Figure 1(b) is that of CRs. Because the minimal zenith angle of Crab in the field of WCDA is $8^{\circ}$, the effective area from $0^{\circ}$ to $8^{\circ}$ in zenith angle is extrapolated. The effective area of any particular energy and zenith can be obtained by interpolation method.

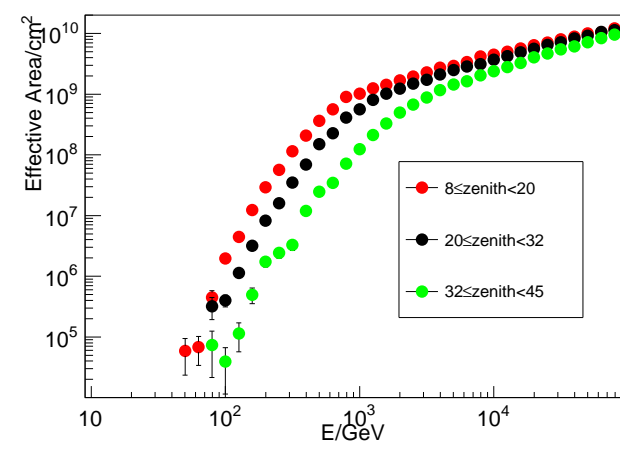

(a)

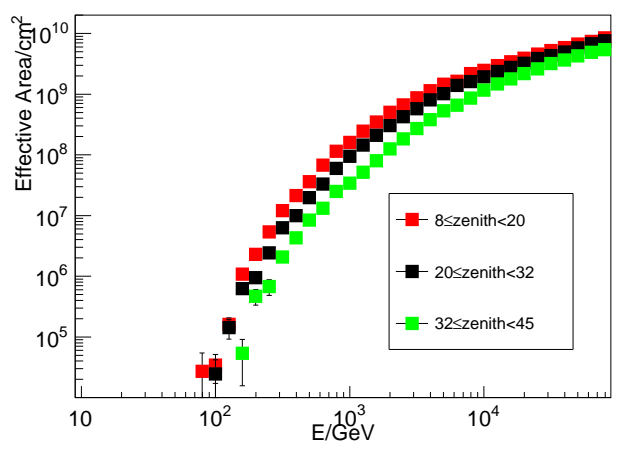

(b)

Figure 1: The $\gamma$-ray(Left) and CRs(right) effective area of WCDA. They increase with energy in different zenith ranges. Red dots denote the $8^{\circ} \sim 20^{\circ}$ zenith angle range; black dots denote the $20^{\circ} \sim 32^{\circ}$ zenith angle range; green dots denote the $32^{\circ} \sim 45^{\circ}$ zenith angle range.

The angular resolution describes the difference between the original and reconstructed directions. When simulating particles, we have to sample the reconstructed direction properly. Figure 2 shows the angular resolution of this paper which agrees well with the Crab-center simulation in [5]. The optimized smooth angular radius is $0.56^{\circ}$ ( $58 \%$ containment) via maximizing the significance of point source.

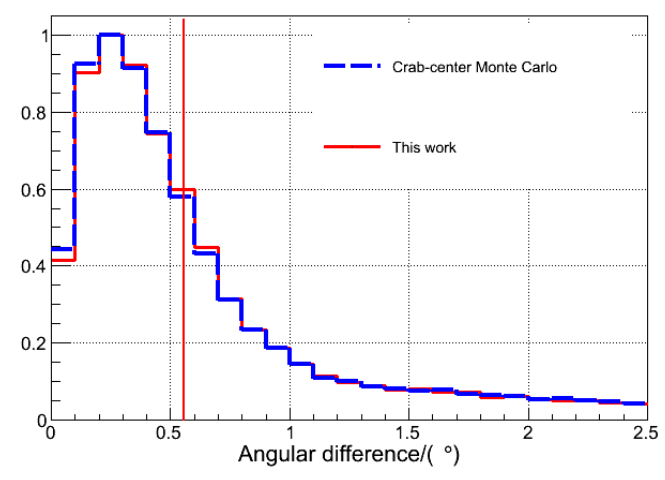

Figure 2: Angular resolution of two simulations. The blue dashed line is the angular resolution from the Crab-center simulation[5], and the red solid line is the angular resolution in this work. The results of two simulations agree well. The red vertical line denotes the optimized angular radius $\left(0.56^{\circ}\right)$ to maximize the significance for point sources.

WCDA is capabale of distinguishing the $\gamma$ rays form CRs using the Muon information. The CRs are tend to generate more Muon with large lateral momentum, therefore, a large signal far away from the core is implies more likely a CRs' siganal, vice looks more like a $\gamma$-ray event. A parameter compactness is used to quantify this character. The compactness is defined as the 
$n P M T s / C x P E_{45}$ where $C x P E_{45}$ is the number of photo-electrons (PEs) in the PMT with the largest signal that is located outside a radius of 45 meters from the reconstructed air shower core. We select the data passing the conditions that the number of fired PMTs is greater than 128 and the zenith angle is smaller than $45^{\circ}$. For this set of data, the optimized photon/hadron discrimination criterion is compactness $>14.4$, where the surviving ratio of $\gamma$ rays $\left(\eta_{\gamma}\right)$ and $\operatorname{CRs}\left(\eta_{c r}\right)$ is $40 \%$ and $0.27 \%$ respectively. This criterion is based on [5].

\section{Fast Simulation}

Different from the Crab simulation, this work predicts the significance of all sources in TevCat, so that a fast simulation is carried out. In this work, FOV of WCDA is from zenith $0^{\circ}$ to $45^{\circ}$. We project the FOV in a zenith-azimuth two-dimension coordinates system, in which the zenith angle is binned in $0.08^{\circ}$-angle bins and the azimuth is binned in $\frac{0.08^{\circ}}{\sin \theta}$-angle bins, so that each pixel owns the same solid angle $\Omega=1.95 \times 10^{-6}$. At the same time, a sidereal day is divided into 3600 time bins, in other words, one day contains 3600 pictures with exposure time of 24 seconds. Each picture records the number of events in horizontal coordinate. The predicted number of cosmic rays and diffuse $\gamma$-ray events is calculated as

$$
N_{i}(t, \theta, \phi)=\int_{E} \phi_{i}(E) A_{i}(\theta, E) \Omega d E \eta_{i} \delta t
$$

where $\Omega$ is the solid angle; $\delta t$ is the time span of one picture that is 24 seconds. When $i$ denotes the CRs, $A_{i}(\theta, E)$ is the differential effective area of cosmic rays; $\phi_{i}(E)$ is the cosmic ray spectrum in [6]; $\eta_{i}$ is the surviving ratio of CRs which passed the $\gamma / P$ criterion. When $i$ denotes the diffuse $\gamma$ rays, these parameters are values of $\gamma$ rays. Among them, the diffuse $\gamma$ rays flux is calculated using GALPROP according to the model in paper [7] extending to $100 \mathrm{TeV}$. The predicted number of point-like $\gamma$-ray events is calculated as

$$
N_{\gamma}(t, \theta, \phi)=\int_{E} \phi(E)_{\gamma} A_{\gamma}(\theta, E) d E \eta_{\gamma} \delta t
$$

where the Crab $\gamma$-ray flux is measured by HEGRA [8] and spectrum information of other sources are listed in the Table1,2,3,4. The meaning of each parameter is the same as (3.1), but represents the property of $\gamma$-ray. We track every source located in the FOV and calculate the number of $\gamma$ ray events from each source. The detected direction of events from each point-like source should be dealt with a point spread function derived from Figure 2 to simulate the angular resolution of WCDA. Finally, we sum up $\gamma$ rays and CRs events in each pixel.

\section{Results}

To analysis the significance of point sources, the All-Distance Equi-Zenith Angle Method is used. It is assumed that the detect efficency do not depend on the azimuth angle, so the background can be estimated from the sideband of the same zenith angle. It has been proved to be effective in [9].

Based on the spectra in TeVCat, Figure 3 shows the significance of all known TeV-sources.Although the significances of a lot of sources are greater than 12, Figure 3 limits the range from -5 to 12 
for visualization. The detailed information are presented in Table 1,2,3,4. Among the observed sources with significance greater than $5,64 \%$ are galactic sources, mainly constituting of unidentified sources. The other $36 \%$ are extragalactic sources which are all AGNs. This result is consistent with the prediction of AGNs in paper[10].

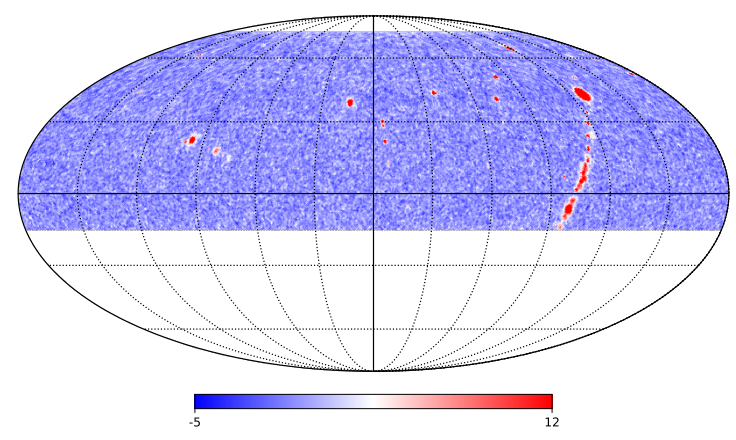

Figure 3: The significance of all TeV-sources.

Table 1: Significance of SNRs, Shells and Binaries: $\sigma$ is the significance of sources, $N_{0}$ is the differential flux at $E_{0}, \beta$ is the spectral index, Extend is the extended angular radius in degree, R.A. is right ascension, Dec is declination.

\begin{tabular}{lllllllll} 
TeVCat NAME & $\begin{array}{c}\text { R.A. } \\
\left(^{\circ}\right)\end{array}$ & $\begin{array}{c}\text { Dec. } \\
\left(^{\circ}\right)\end{array}$ & $\sigma$ & $\begin{array}{c}N_{0} \\
\left(\mathrm{TeV}^{-1} \mathrm{~cm}^{-2} \mathrm{~s}^{-1}\right)\end{array}$ & $\begin{array}{c}E_{0} \\
(\mathrm{TeV})\end{array}$ & $\begin{array}{c}\text { Extend } \\
\left({ }^{\circ}\right)\end{array}$ & $\begin{array}{l}\text { Ref. } \\
\text { ARGOJ2031+4157 }\end{array}$ \\
\hline Ho7.8 & 42.50 & 151.89 & $3.50 \mathrm{E}-09$ & 0.1 & 2.16 & 2 & {$[11]$} \\
W49B & 287.78 & 9.16 & 11.86 & $3.15 \mathrm{E}-13$ & 1 & 3.14 & - & {$[12]$} \\
CassiopeiaA & 350.81 & 58.81 & 7.22 & $1.45 \mathrm{E}-12$ & 1 & 2.75 & - & {$[13]$} \\
HESSJ1912+101 & 288.20 & 10.15 & 10.17 & $3.66 \mathrm{E}-14$ & 7 & 2.64 & 0.7 & {$[2]$} \\
W51 & 290.73 & 14.19 & 8.48 & $2.61 \mathrm{E}-14$ & 7 & 2.51 & 0.9 & {$[2]$} \\
HESSJ1848-018 & 282.12 & -1.79 & 5.18 & $3.70 \mathrm{E}-12$ & 1 & 2.8 & 0.32 & {$[14]$} \\
LSI+61303 & 40.14 & 61.26 & 10.17 & $1.80 \mathrm{E}-12$ & 1 & 2.34 & - & {$[15]$} \\
HESSJ1832-093 & 278.21 & -9.38 & 10.29 & $4.80 \mathrm{E}-13$ & 1 & 2.6 & - & {$[16]$} \\
SNRG015.4+00.1 & 274.52 & -15.47 & 7.55 & $9 \mathrm{E}-12$ & 1.9 & 2.3 & - & {$[17]$} \\
\hline \hline \multicolumn{7}{c}{$a:$ The spectrum of this source exhibits a cutoff at the energy of 40TeV. }
\end{tabular}


Table 2: Significance of PWNe: $\sigma$ is the significance of sources, $N_{0}$ is the differential flux at $E_{0}, \beta$ is the spectral index, Extend is the extended angular radius in degree, R.A. is right ascension, Dec is declination.

\begin{tabular}{|c|c|c|c|c|c|c|c|c|}
\hline TeVCat NAME & $\begin{array}{c}\text { R.A. } \\
\left({ }^{\circ}\right)\end{array}$ & $\begin{array}{l}\text { Dec. } \\
\left({ }^{\circ}\right)\end{array}$ & $\sigma$ & $\begin{array}{c}N_{0} \\
\left(\mathrm{TeV}^{-1} \mathrm{~cm}^{-2} \mathrm{~s}^{-1}\right)\end{array}$ & $\begin{array}{c}E_{0} \\
(\mathrm{TeV})\end{array}$ & $\beta$ & $\begin{array}{c}\text { Extend } \\
\left({ }^{\circ}\right)\end{array}$ & Ref. \\
\hline TeV2032+4130 & 308.03 & 41.51 & 150.48 & $6.16 \mathrm{E}-14$ & 7 & 2.52 & 0.7 & [2] \\
\hline SNRG054.1+00.3 & 292.63 & 18.87 & 21.83 & $9.80 \mathrm{E}-15$ & 7 & 2.74 & - & [2] \\
\hline HESSJ1833-105 & 278.39 & -10.56 & 6.60 & $4.59 \mathrm{E}-13$ & 1 & 2.08 & _ & [18] \\
\hline HESSJ1831-098 & 277.85 & -9.90 & 9.60 & $9.58 \mathrm{E}-14$ & 7 & 2.64 & 0.9 & [2] \\
\hline Geminga & 98.12 & 17.37 & 10.69 & 4.87E-14 & 7 & 2.23 & 2 & [2] \\
\hline Crab & 83.63 & 22.01 & 307.66 & $1.85 \mathrm{E}-13$ & 7 & 2.58 & - & [2] \\
\hline
\end{tabular}

Table 3: Significance of AGNs: $\sigma$ is the significance of sources, $N_{0}$ is the differential flux at $E_{0}, \beta$ is the spectral index, $E_{\text {cut }}$ is cutoff energy, R.A. is right ascension, Dec is declination.

\begin{tabular}{lllllllll} 
TeVCat NAME & $\begin{array}{c}\text { R.A. } \\
\left({ }^{\circ}\right)\end{array}$ & $\begin{array}{c}\text { Dec. } \\
\left({ }^{\circ}\right)\end{array}$ & $\sigma$ & $\begin{array}{c}N_{0} \\
\left(\mathrm{TeV}^{-1} \mathrm{~cm}^{-2} \mathrm{~s}^{-1}\right)\end{array}$ & $\begin{array}{c}E_{0} \\
(\mathrm{TeV})\end{array}$ & $\begin{array}{c}E_{\text {cut }} \\
(\mathrm{TeV})\end{array}$ & Ref. \\
\hline 1ES1959+650 & 300.00 & 65.15 & 105.61 & $3.05 \mathrm{E}-11$ & 1 & 2.79 & - & {$[19]$} \\
RGBJ0710+591 & 107.61 & 59.15 & 5.84 & $9.20 \mathrm{E}-13$ & 1 & 2.69 & - & {$[20]$} \\
1ES2344+514 & 356.77 & 51.71 & 19.17 & $2.65 \mathrm{E}-12$ & 0.91 & 2.46 & - & {$[21]$} \\
RGBJ2056+496 & 314.18 & 49.67 & 9.68 & $1.15 \mathrm{E}-11$ & 0.4 & 2.77 & - & {$[22]$} \\
3C66A & 35.67 & 43.04 & 7.86 & $9.60 \mathrm{E}-11$ & 0.2 & 3.64 & - & {$[23]$} \\
H1426+428 & 217.14 & 42.67 & 61.57 & $4.37 \mathrm{E}-12$ & 1 & 3.54 & - & {$[24]$} \\
Markarian501 & 253.47 & 39.76 & 47.50 & $4.40 \mathrm{E}-12$ & 1 & 1.6 & 5.7 & {$[2]$} \\
Markarian421 & 166.08 & 38.19 & 236.84 & $2.82 \mathrm{E}-11$ & 1 & 2.21 & 5.4 & {$[2]$} \\
1ES1218+304 & 185.36 & 30.19 & 17.85 & $1.40 \mathrm{E}-12$ & 1 & 3.13 & - & {$[25]$} \\
1ES1215+303 & 184.45 & 30.10 & 9.72 & $2.30 \mathrm{E}-11$ & 0.3 & 3.6 & - & {$[26]$} \\
WComae & 185.38 & 28.23 & 11.05 & $2.00 \mathrm{E}-11$ & 0.4 & 3.81 & - & {$[27]$} \\
VERJ0521+211 & 80.44 & 21.21 & 12.44 & $1.99 \mathrm{E}-11$ & 0.4 & 3.44 & - & {$[28]$} \\
PG1553+113 & 238.94 & 11.19 & 6.33 & $4.80 \mathrm{E}-11$ & 0.3 & 4.33 & - & {$[29]$} \\
S30218+35 & 35.27 & 35.94 & 6.43 & $2.00 \mathrm{E}-09$ & 0.1 & 3.8 & - & {$[30]$} \\
4C+21.35 & 186.23 & 21.38 & 32.17 & $7.80 \mathrm{E}-10$ & 0.2 & 3.75 & - & {$[31]$} \\
M87 & 187.70 & 12.40 & 6.82 & $7.70 \mathrm{E}-12$ & 0.3 & 2.21 & - & {$[32]$} \\
RXJ0648.7+1516 & 102.19 & 15.27 & 5.21 & $2.30 \mathrm{E}-11$ & 0.3 & 4.4 & - & {$[33]$} \\
1ES1727+502 & 262.08 & 50.22 & 39.73 & $7.8 \mathrm{E}-12$ & 0.62 & 2.1 & - & {$[34]$} \\
\hline \hline
\end{tabular}


Table 4: Significance of unidentified sources(UID): $\sigma$ is the significance of sources, $N_{0}$ is the differential flux at $E_{0}, \beta$ is the spectral index, Extend is the extended angular radius in degree, R.A. is right ascension, Dec is declination.

\begin{tabular}{|c|c|c|c|c|c|c|c|c|}
\hline TeVCat NAME & $\begin{array}{l}\text { R.A. } \\
\left({ }^{\circ}\right)\end{array}$ & $\begin{array}{c}\text { Dec. } \\
\left({ }^{\circ}\right)\end{array}$ & $\sigma$ & $\begin{array}{c}N_{0} \\
\left(\mathrm{TeV}^{-1} \mathrm{~cm}^{-2} \mathrm{~s}^{-1}\right)\end{array}$ & $\begin{array}{c}E_{0} \\
(\mathrm{TeV})\end{array}$ & $\beta$ & $\begin{array}{c}\text { Extend } \\
\left({ }^{\circ}\right)\end{array}$ & Ref. \\
\hline MAGICJ0223+403 & 35.80 & 43.01 & 7.42 & $9.60 \mathrm{E}-11$ & 0.2 & 3.64 & - & [23] \\
\hline MGROJ2031+41 & 307.18 & 41.31 & 6.82 & $2.10 \mathrm{E}-14$ & 10 & 3.22 & 1.8 & [35] \\
\hline VERJ2019+407 & 305.02 & 40.76 & 22.93 & $1.50 \mathrm{E}-12$ & 1 & 2.37 & 0.23 & [36] \\
\hline 2HWCJ2006+341 & 301.55 & 34.18 & 17.11 & $9.60 \mathrm{E}-15$ & 7 & 2.64 & - & [2] \\
\hline 2HWCJ1953+294 & 298.26 & 29.48 & 21.77 & $8.30 \mathrm{E}-15$ & 7 & 2.78 & - & [2] \\
\hline 2HWCJ1955+285 & 298.83 & 28.59 & 8.14 & $5.70 \mathrm{E}-15$ & 7 & 2.4 & - & [2] \\
\hline 2HWCJ1938+238 & 294.74 & 23.81 & 26.00 & $7.40 \mathrm{E}-15$ & 7 & 2.96 & - & [2] \\
\hline 2HWCJ1928+177 & 292.15 & 17.78 & 19.31 & $1.07 \mathrm{E}-14$ & 7 & 2.6 & - & [37] \\
\hline 2HWCJ1921+131 & 290.30 & 13.13 & 20.28 & $7.90 \mathrm{E}-15$ & 7 & 2.75 & - & [2] \\
\hline 2HWCJ1914+117 & 288.68 & 11.72 & 20.73 & $8.50 \mathrm{E}-15$ & 7 & 2.83 & - & [2] \\
\hline 2HWCJ1907+084 & 286.79 & 8.50 & 32.00 & $7.30 \mathrm{E}-15$ & 7 & 3.25 & - & [2] \\
\hline 2HWCJ1829+070 & 277.34 & 7.03 & 11.06 & $8.10 \mathrm{E}-15$ & 7 & 2.69 & - & [2] \\
\hline MGROJ1908+06 & 286.98 & 6.27 & 13.44 & $8.51 \mathrm{E}-14$ & 7 & 2.33 & 0.8 & [2] \\
\hline 2HWCJ1902+048 & 285.51 & 4.86 & 29.55 & $8.30 \mathrm{E}-15$ & 7 & 3.22 & - & [2] \\
\hline MAGICJ1857.6+029? & 7284.40 & 2.97 & 10.55 & $6.10 \mathrm{E}-12$ & 1 & 2.39 & 0.1 & [38] \\
\hline HESSJ1858+020 & 284.58 & 2.09 & 6.90 & $6.00 \mathrm{E}-13$ & 1 & 2.17 & 0.08 & [38] \\
\hline 2HWCJ1852+013 & 283.01 & 1.38 & 24.02 & $1.82 \mathrm{E}-14$ & 7 & 2.9 & - & [2] \\
\hline 2HWCJ1844-032 & 281.07 & -3.25 & 11.25 & $9.28 \mathrm{E}-14$ & 7 & 2.51 & 0.6 & [2] \\
\hline 2HWCJ1309-054 & 197.31 & -5.49 & 7.75 & $1.23 \mathrm{E}-14$ & 7 & 2.55 & - & [2] \\
\hline 2HWCJ1837-065 & 279.36 & -6.58 & 36.28 & $3.41 \mathrm{E}-13$ & 7 & 2.66 & 2 & [2] \\
\hline HESSJ1834-087 & 278.69 & -8.76 & 7.93 & $3.70 \mathrm{E}-12$ & 1 & 2.5 & 0.14 & [37] \\
\hline HESSJ1813-126 & 273.34 & -12.69 & 5.86 & $2.74 \mathrm{E}-14$ & 7 & 2.84 & - & [2] \\
\hline 2HWCJ1825-134 & 276.46 & -13.40 & 7.55 & $2.49 \mathrm{E}-13$ & 7 & 2.56 & 0.9 & [2] \\
\hline
\end{tabular}




\section{Discussion}

Pulsar Wind Nebulae(PWNe) are the most common Galactic TeV sources. A central rotating neutron star powers an outflow of energetic electrons: the pulsar wind. Some arguments presume that local source may explain the excess of the positron, however, the results of HAWC about gamma-halo around Geminga and Monogem indicate a very slow diffusion of cosmic rays which contributes too small local flux to explain the excess of positron[39]. A scenario of two-zone diffusion model that the diffusion is slow only in a small region around the source, while on the outside the diffusion is fast as usual is proposed in [40]. Considering the large field of view and high sensitivity of WCDA, it will preforms a better observation of extended sources in contrast to IACTs and contribute to the understanding of cosmic ray propagation around sources.

In addition, blazar emission can be extremely variable with flares up to ten times the quiescent flux or more. When a source is on an flaring state and its flux would be enhanced by a factor of N, the time required to detect the source at fixed significance would be reduced by $N^{2}$. WCDA would be capable observing more flares in the context that the spectrum adopted in this paper is similar to stable state. WCDA also has advantages to discover unexpected other blazar flares and provide alerts to IACTs for follow-up observation.

As we have mentioned in Section 2, the design of WCDA has been modified to 3 subarrays. One large subarray measuring $300 m \times 110 m$ will replace two subarrays. Moreover, 8 -in PMTs will be changed to 20 -in PMTs in order to improve the sensitivity at $\sim 100 \mathrm{GeV}$. Since our analysis is performed at a higher energy, such differences would not change our result significantly. Due to the improvement of the sensitivity at lower energy, WCDA will give a better constraint on the intensity of extragalactic background light(EBL)[10] by the observed spectra of AGNs.

\section{Acknowledgments}

This work is supported by the National Key $R \& D$ Program of China:2018YFA0404202,11761141001, $11635011,11873005$.

\section{References}

[1] J K Becker, W Bednarek, K Berger, et al. ApJ, 664:L91-L94, 2007.

[2] A. U. Abeysekara, A. Albert, R. Alfaro, et al. ApJ, 841:100, 2017.

[3] A. U. Abeysekara, A. Albert, R. Alfaro, et al. ApJ, 843(1):39, 2017.

[4] A. U. Abeysekara, A. Albert, R. Alfaro, et al. Nature, 562(7725):82-85, 2018.

[5] Zhiguo Yao and et al. In https://galprop.stanford.edu/elibrary/icrc/2009/preliminary/pdf/icrc0720.pdf, 2007.

[6] T. K. Gaisser, T. Stanev, and S. Tilav. Front. Phys., 8(6):748-758, 2013.

[7] Yi Qing Guo, Hong Bo Hu, and Zhen Tian. Chinese Physics C, 40(11):1-8, 2016.

[8] B. Bartoli, P. Bernardini, X. J. Bi, et al. ApJ, 798:119, 2015.

[9] M. Amenomori, S. Ayabe, D. Chen, et al. The Astrophysical Journal, 633:1005-1012, 2005. 
[10] Yi Zhao, Qiang Yuan, Xiao-Jun Bi, Feng-Rong Zhu, and Huan-Yu Jia. International Journal of Modern Physics D, 25(01):1650006, 2016.

[11] B. Bartoli, P. Bernardini, X. J. Bi, et al. ApJ, 790:152, 2014.

[12] H. E. S. S. Collaboration. A\&A, 612:A5, 2018.

[13] Sajan Kumar. In Proceedings of Science, 2015.

[14] R. C.G. Chaves, M. Renaud, M. Lemoine-Goumard, and P. Goret. In AIP Conference Proceedings, volume 1085, pages 372-375, 2009.

[15] S. Archambault, A. Archer, T. Aune, et al. ApJL, 817:L7, 2016.

[16] A. Abramowski, F. Acero, F. Aharonian, et al. Monthly Notices of the Royal Astronomical Society, 446:1163-1169, 2015.

[17] HESS Collaboration. 562:1-10, 2013.

[18] H. Djannati-Atai, OC De Jager, R. Terrier, YA Gallant, and S. Hoppe. In Proceedings ofthe 30th International Cosmic Ray Conference, volume 2, pages 823-826, 2008.

[19] M. Santander. In Proceedings of Science, pages 1-7, 2017.

[20] V. A. Acciari, E. Aliu, T. Arlen, et al. ApJL, 715:L49-L55, 2010.

[21] C. Allen, S. Archambault, A. Archer, et al. Monthly Notices of the Royal Astronomical Society, 471:2117-2123, 2017.

[22] Wystan Benbow. In Proceedings of Science, 2017.

[23] J. Aleksić, L. A. Antonelli, P. Antoranz, et al. ApJ, 726:58, 2011.

[24] D. Petry, I.H. Bound, S. M. Bradbury, et al. ApJ, 580:104-109, 2002.

[25] Veritas Collaboration. In 33RD INTERNATIONAL COSMIC RAY CONFERENCE, RIO DE JANEIRO 2013, pages 1-4, 2013.

[26] E Aliu, S Archambault, T Arlen, et al. ApJ, 779:92, 2013.

[27] V.A. Acciari, E. Aliu, M. Beilicke, W. Benbow, and S. M. Bradbury. ApJ, 684:L73-L77, 2008.

[28] S Archambault, T Arlen, T Aune, et al. APJ, 776(69):(10pp), 2013.

[29] E Aliu, A Archer, T Aune, et al. ApJ, 799:7, 2015.

[30] M L Ahnen, S Ansoldi, L A Antonelli, et al. A\&A, 595:A98, 2016.

[31] L A Antonelli, P Antoranz, M Backes, et al. ApJL, 730:L8, 2011.

[32] J Aleksic, E.A. Alvarez, L. A. Antonelli, P. Antoranz, and M. Asensio. A\&A, 544:A96, 2012.

[33] E Aliu, T Aune, M Beilicke, et al. ApJ, 127:1-7, 2011.

[34] S Archambault, A Archer, M Beilicke, et al. 808(110):11, 2015.

[35] A. A. Abdo, U. Abeysekara, B. T. Allen, et al. ApJ, 753:159, jul 2012.

[36] E. Aliu, S. Archambault, T. Arlen, et al. ApJ, 770:93, 2013.

[37] D Cornwall and A Mattingly. ApJ, 643:L53-L56, 2006.

[38] F Aharonian, A G Akhperjanian, U Barres De Almeida, B Behera, and M Beilicke. A\&A, 477:353-363, 2008.

[39] A. U. Abeysekara, A. Albert, R. Alfaro, et al. Science, 358(6365):911-914, 2017.

[40] Kun Fang, Xiao-Jun Bi, Peng-Fei Yin, and Qiang Yuan. 2018. 